\title{
THE UNICITY OF BEST APPROXIMATION IN A SPACE OF COMPACT OPERATORS
}

\author{
JOANNA KOWYNIA*
}

\begin{abstract}
Chebyshev subspaces of $\mathscr{K}\left(c_{0}, c_{0}\right)$ are studied. A $k$-dimensional non-interpolating Chebyshev subspace is constructed. The unicity of best approximation in non-Chebyshev subspaces is considered.
\end{abstract}

\section{Introduction}

Let $\mathrm{K}$ be the field of real or complex numbers and let $(X,\|\cdot\|)$ be a normed space over K. Let ext $S_{X^{\star}}$ denote the set of all extreme points of $S_{X^{\star}}$, where $S_{X^{\star}}$ is the unit sphere in $X^{\star}$.

For every $x \in X$ we put

$$
E(x)=\left\{f \in \operatorname{ext} S_{X^{\star}}: f(x)=\|x\|\right\} .
$$

By the Hahn-Banach and the Krein-Milman Theorems, $E(x) \neq \emptyset$.

Let for $Y \subset X$,

$$
P_{Y}(x)=\{y \in Y:\|x-y\|=\operatorname{dist}(x, Y)\} .
$$

A linear subspace $Y \subset X$ is called $a$ Chebyshev subspace if for every $x \in X$ the set $P_{Y}(x)$ contains one and only one element.

Theorem 1 (see [3]). Assume $X$ is a normed space, $Y \subset X$ is a linear subspace, and let $x \in X \backslash Y$. Then $y_{0} \in P_{Y}(x)$ if and only if for every $y \in Y$ there exists $f \in E\left(x-y_{0}\right)$ with $\Re f(y) \leq 0$.

Definition (see, e.g., [8]). An element $y_{0} \in Y$ is called a strongly unique best approximation for $x \in X$ if there exists $r>0$ such that for every $y \in Y$,

$$
\|x-y\| \geq\left\|x-y_{0}\right\|+r\left\|y-y_{0}\right\| .
$$

\footnotetext{
* Research supported by local grant No. 10.420.03.

Received 29 June 2009.
} 
The biggest constant $r$ satisfying the above inequality is called $a$ strong unicity constant. There exist two main applications of a strong unicity constant:

- the error estimate of the Remez algorithm (see e.g. [13]),

- the Lipschitz continuity of the best approximation mapping at $x_{0}$ (assuming that there exists a strongly unique best approximation to $x_{0}$ ) (see e.g. [5], [9], [11]).

Theorem 2 (see [17]). Let $x \in X \backslash Y$ and let $Y$ be a linear subspace of $X$. Then $y_{0} \in Y$ is a strongly unique best approximation for $x$ with a constant $r>0$ if and only if for every $y \in Y$ there exists $f \in E\left(x-y_{0}\right)$ with $\Re f(y) \leq-r\|y\|$.

Recall that a $k$-dimensional subspace $\mathscr{V}$ of a normed space $X$ is called $a n$ interpolating subspace if for any linearly independent $f_{1}, f_{2}, \ldots, f_{k} \in \operatorname{ext} S_{X^{\star}}$ and for every $v \in \mathscr{V}$ the following holds:

$$
\text { if } f_{i}(v)=0, i=1,2, \ldots, k \text { then } v=0 .
$$

Every interpolating subspace is a finite dimensional Chebyshev subspace. If $\mathscr{V} \subset X$ is an interpolating subspace then every $x \in X$ has a strongly unique best approximation in $\mathscr{V}$ (see [2]).

In this paper we consider $X=\mathscr{K}\left(c_{0}, c_{0}\right)$ (the space of all compact operators from $c_{0}$ to $c_{0}$ equipped with the operator norm). Here $c_{0}$ denotes the space of all real sequences convergent to zero. For any $x=\left(x_{k}\right) \in c_{0}$ we put

$$
\|x\|_{\infty}=\sup _{k}\left|x_{k}\right| .
$$

In [8, Theorem 3.1] it has been proved that if $\mathscr{V} \subset \mathscr{K}\left(c_{0}, c_{0}\right)$ is a finitedimensional Chebyshev subspace then every $A \in \mathscr{K}\left(c_{0}, c_{0}\right)$ has a strongly unique best approximation in $\mathscr{V}$. However, in [8] no example of a non-interpolating Chebyshev subspace has been proposed. If it were true that any finitedimensional Chebyshev subspace of $\mathscr{K}\left(c_{0}, c_{0}\right)$ is an interpolating subspace we would have obtained the proof of Theorem 3.1, [8] immediately (see [2] for more details).

The aim of this paper is to show that for every $k<\infty$ there exists a $k$ dimensional non-interpolating Chebyshev subspace of $\mathscr{K}\left(c_{0}, c_{0}\right)$. This result is quite different from the result obtained in [7]. In the space $\mathscr{L}\left(l_{1}^{n}, c_{0}\right)$ any finite-dimensional Chebyshev subspace is an interpolating subspace.

Additionally, we discuss the strong unicity of best approximation in some (not necessarily Chebyshev) subspaces of $\mathscr{K}\left(c_{0}, c_{0}\right)$. 


\section{2. $\boldsymbol{k}$-dimensional Chebyshev subspaces of $\mathscr{K}\left(c_{0}, c_{0}\right)$}

Let $A \in \mathscr{K}\left(c_{0}, c_{0}\right)$ be represented by a matrix $\left[a_{i j}\right]_{i, j \in \mathrm{N}}$. Note that

$$
\left(a_{i j}\right)_{i=1}^{\infty} \in c_{0} \quad \text { for every } \quad j \in \mathrm{N} .
$$

Since each row of a matrix $\left[a_{i j}\right]_{i, j \in \mathrm{N}}$ corresponds to a linear functional on $c_{0}$,

$$
\left(a_{i j}\right)_{j=1}^{\infty} \in l^{1} \quad \text { for every } \quad i \in \mathrm{N} .
$$

Moreover, by the Schur Theorem (see [6])

$$
\lim _{i \rightarrow \infty}\left(\sum_{j=1}^{\infty}\left|a_{i j}\right|\right)=0 .
$$

Recall (see [4]) that ext $S_{\mathscr{K}^{*}\left(c_{0}, c_{0}\right)}$ consists of functionals of the form $e_{i} \otimes x$, where $x \in \operatorname{ext} S_{l \infty}$ and

$$
\left(e_{i} \otimes x\right)(A)=\sum_{j=1}^{\infty} x_{j} a_{i j}
$$

It is easy to see that

$$
\|A\|=\sup _{i \geq 1} \sum_{j=1}^{\infty}\left|a_{i j}\right| .
$$

REMARK 1. Let $X$ be a Banach space and let $\mathscr{V}$ be a finite-dimensional subspace with $V_{1}, V_{2}, \ldots, V_{k}$ as a basis. Then $\mathscr{V}$ is an interpolating subspace if and only if for any linearly independent $f_{1}, f_{2}, \ldots, f_{k} \in$ ext $S_{X^{\star}}$ the determinant of $\left[f_{i}\left(V_{j}\right)\right]_{i, j=1,2, \ldots, k}$ is not equal to zero.

Proof. We apply the definition of a $k$-dimensional interpolating subspace and the theory of linear equations. This completes the proof.

In the sequel, we denote by $\operatorname{lin}\left\{V_{1}, V_{2}, \ldots, V_{k}\right\}$ the $k$-dimensional subspace of $\mathscr{K}\left(c_{0}, c_{0}\right)$ with $V_{1}, V_{2}, \ldots, V_{k}$ as a basis.

Example 1. Let $V=\left[v_{i j}\right]_{i, j \in \mathrm{N}}$, where $v_{i 1}=\frac{1}{2^{i}}, v_{i j}=0, i, j \in \mathrm{N}, j \geq 2$. It is obvious that $\mathscr{V}=\operatorname{lin}\{V\}$ is a one-dimensional interpolating subspace of $\mathscr{K}\left(c_{0}, c_{0}\right)$. 
Theorem 3. Let $\mathscr{V}=\operatorname{lin}\left\{V_{1}, V_{2}, \ldots, V_{n}\right\}$. Let $V_{m}=\left[\left(v_{m}\right)_{i j}\right]_{i, j \in \mathrm{N}}, m=$ $1,2, \ldots, n$. If $\mathscr{V}$ is a Chebyshev subspace then

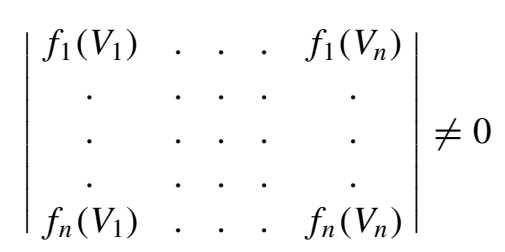

for any $f_{1}, \ldots, f_{n} \in \operatorname{ext} S_{\mathscr{r}^{*}\left(c_{0}, c_{0}\right)}$ such that $f_{m}=e_{i_{m}} \otimes x^{i_{m}}, m=1,2, \ldots, n$, where $i_{m} \neq i_{k}$ for $m \neq k$.

Proof. Assume (3) does not hold. Therefore there exist $f_{1}, \ldots, f_{n} \in$ ext $S_{\mathscr{K} *\left(c_{0}, c_{0}\right)}, f_{m}=e_{i_{m}} \otimes x^{i_{m}}, m=1,2, \ldots, n$, where $i_{m} \neq i_{k}$ for $m \neq k$ such that $\operatorname{det} D=0$, where

$$
D=\left[\begin{array}{ccccc}
f_{1}\left(V_{1}\right) & \cdot & \cdot & \cdot & f_{1}\left(V_{n}\right) \\
\cdot & \cdot & \cdot & \cdot & \cdot \\
\cdot & \cdot & \cdot & \cdot & \cdot \\
\cdot & \cdot & \cdot & \cdot & \cdot \\
f_{n}\left(V_{1}\right) & \cdot & \cdot & \cdot & f_{n}\left(V_{n}\right)
\end{array}\right] .
$$

Since $\operatorname{det} D=\operatorname{det} D^{T}$, there exists $y=\left(y_{1}, y_{2}, \ldots, y_{n}\right) \neq 0$ such that $D^{T} y=$ 0 . Consequently,

$$
\left.\sum_{m=1}^{n} y_{m} f_{m}\right|_{\mathscr{V}}=0 .
$$

Since $y \neq 0$, replacing $f_{m}$ by $-f_{m}$ if necessary, we may assume $y_{m} \geq 0$ for $m=1,2, \ldots, n$ and

$$
\sum_{m=1}^{n} y_{m}=1 \text {. }
$$

Set $\mathscr{C}=\left\{l \in\{1,2, \ldots, n\}: y_{l}>0\right\}$.

Fix $\left(d_{j}\right)_{j \in \mathrm{N}}$ with the following properties:

$$
d_{j}>0, \quad j \in \mathrm{N} \quad \text { and } \quad \sum_{j=1}^{\infty} d_{j}=1 .
$$

Define $A=\left[a_{i_{p} j}\right]_{i_{p}, j \in \mathrm{N}} \in \mathscr{K}\left(c_{0}, c_{0}\right)$ by

$$
\begin{array}{ll}
a_{i_{p} j}=0 & \text { for } \quad p \notin \mathscr{C}, j \in \mathbf{N}, \\
a_{i_{p} j}=d_{j} \cdot \operatorname{sgn} x^{i_{p}}(j) & \text { for } \quad p \in \mathscr{C}, j \in \mathbf{N} .
\end{array}
$$


Note that $\|A\|=1$ and

$$
E(A)=\left\{f_{p}: p \in \mathscr{C}\right\}
$$

By (4) and Theorem 1, $0 \in \mathscr{P}_{\mathscr{V}}(A)$.

Since det $D=0$, there exists $x=\left(x_{1}, x_{2}, \ldots, x_{n}\right) \neq 0$ such that $D x=0$. Put

$$
V=\sum_{m=1}^{n} x_{m} V_{m} .
$$

Note that $V \neq 0$ and $f_{m}(V)=0, m=1,2, \ldots, n$. By Theorem 2,0 is not a strongly unique best approximation for $A$ in $\mathscr{V}$. By [8, Theorem 3.1], $\mathscr{V}$ is not a Chebyshev subspace and the proof is complete.

Theorem 4. Let $\mathscr{V}=\operatorname{lin}\{V\}, V \in \mathscr{K}\left(c_{0}, c_{0}\right), V \neq 0$. Then $\mathscr{V}$ is a Chebyshev subspace if and only if $\mathscr{V}$ is an interpolating subspace.

Proof. The classical work here is [12]. In $l^{1}$, the one-dimensional subspace $\operatorname{lin}\{v\}$ is Chebyshev iff for every $x \in \operatorname{ext} S_{l \infty}$ the following holds

$$
\sum_{j=1}^{\infty} x(j) v(j) \neq 0 .
$$

Note that for any $x \in c_{0}$ we obtain $V(x)=\left[f_{1}(x), f_{2}(x), \ldots\right]$, where the functionals $f_{i}$ correspond to elements of $l^{1}$.

It is obvious that if for any $j, \operatorname{lin}\left\{f_{j}\right\}$ is not a Chebyshev subspace of $l^{1}$, then $\operatorname{lin}\{V\}$ is not a Chebyshev subspace of $\mathscr{K}\left(c_{0}, c_{0}\right)$. This proves the theorem.

Note that by a result of Malbrock (see [10], Theorem 3.3) each one-dimensional subspace $\mathscr{V}=\operatorname{lin}\{V\} \subset \mathscr{L}\left(c_{0}, c_{0}\right)$ is a Chebyshev subspace iff there exists $\delta>0$ such that

$$
\left|\sum_{j=1}^{\infty} x(j) v_{i j}\right| \geq \delta,
$$

where $|x(j)|=1, j \in \mathbf{N}$.

Corollary. Let $\mathscr{V} \subset \mathscr{K}\left(c_{0}, c_{0}\right)$ be a one-dimensional Chebyshev subspace. Every operator $A \in \mathscr{K}\left(c_{0}, c_{0}\right)$ has a strongly unique best approximation in $\mathscr{V}$.

Proof. Obvious. For more details we refer the reader to [2].

It is clear that (3) is satisfied for any $\mathrm{n}$-dimensional interpolating subspace. However, (3) is not sufficient for an $n$-dimensional $(n \geq 2)$ subspace to be Chebyshev. 
Example 2. Let $\mathscr{V}=\operatorname{lin}\left\{V_{1}, V_{2}\right\}$, where

$$
V_{1}=\left[\begin{array}{ccccc}
1 & 0 & . & . & . \\
\frac{1}{2} & 0 & . & . & . \\
\frac{1}{4} & 0 & . & . & . \\
. & . & . & . & . \\
. & . & . & . & .
\end{array}\right], \quad V_{2}=\left[\begin{array}{ccccc}
1 & 0 & . & . & . \\
\frac{1}{3} & 0 & . & . & . \\
\frac{1}{9} & 0 & . & . & . \\
. & . & . & . & . \\
. & . & . & . & .
\end{array}\right] .
$$

Note that $\mathscr{V}$ satisfies (3). We claim that $\mathscr{V}$ is a non-Chebyshev subspace.

Indeed, define $A=\left[a_{i j}\right]_{i, j \in \mathrm{N}}$ by

$$
a_{12}=100, a_{i j}=0 \quad \text { for each } \quad(i, j) \neq(1,2), i, j \in \mathrm{N} .
$$

It follows that

$$
A-\left(\alpha_{1} V_{1}+\alpha_{2} V_{2}\right)=\left[\begin{array}{ccccc}
-\alpha_{1}-\alpha_{2} & 100 & 0 & . & . \\
-\frac{1}{2} \alpha_{1}-\frac{1}{3} \alpha_{2} & 0 & . & . & . \\
-\frac{1}{4} \alpha_{1}-\frac{1}{9} \alpha_{2} & 0 & . & . & . \\
. & . & . & . & . \\
. & . & . & . & .
\end{array}\right], \quad \alpha_{1}, \alpha_{2} \in \mathbf{R} .
$$

Hence

$$
\|A\|=\left\|A-\left(600 V_{1}-600 V_{2}\right)\right\|=100=\inf _{\alpha_{1}, \alpha_{2} \in \mathrm{R}}\left\|A-\left(\alpha_{1} V_{1}+\alpha_{2} V_{2}\right)\right\| .
$$

THEOREM 5. Let $V_{1}, V_{2}, \ldots, V_{n}$ be given by

$$
V_{j}=\left[\begin{array}{cccccccc}
0 & 0 & . & . & v_{1 j} & 0 & . & . \\
0 & 0 & . & . & v_{2 j} & 0 & . & . \\
0 & 0 & . & . & v_{3 j} & 0 & . & . \\
. & . & . & . & . & & \\
. & . & . & . & . & & & \\
. & . & . & . & . & & & \\
. & . & . & . & . & & &
\end{array}\right],
$$

where $v_{i j} \neq 0$ for each $i \in \mathrm{N}, j \in\{1,2, \ldots, n\}$ and

$$
\lim _{i \rightarrow \infty} v_{i j}=0 \quad \text { for each } j \in\{1,2, \ldots, n\} .
$$

The following statements are equivalent:

(i) For every choice of distinct $j_{1}, \ldots, j_{k}$ from $\{1,2, \ldots, n\}, \mathscr{V}\left(j_{1}, \ldots, j_{k}\right)$ $:=\operatorname{lin}\left\{V_{j_{1}}, \ldots, V_{j_{k}}\right\}$ is a Chebyshev subspace of $\mathscr{K}\left(c_{0}, c_{0}\right)$ 
(ii)

$$
\begin{gathered}
\forall 1 \leq k \leq n, \quad \forall 1 \leq j_{1}<j_{2}<\cdots<j_{k} \leq n, \\
\forall 1 \leq i_{1}<i_{2}<\cdots<i_{k}, \\
\forall x_{m l} \in \mathrm{R}:\left|x_{m l}\right|=1, \quad m, l=1,2, \ldots, k \\
\operatorname{det}\left[x_{m l} v_{i_{m} j_{l}}\right]_{m=1,2, \ldots, k, l=1,2, \ldots, k} \neq 0 .
\end{gathered}
$$

Proof. First, we assume that (ii) holds.

If $k=1$ then $\mathscr{V}\left(j_{1}\right)$ is an interpolating subspace for every $j_{1} \in\{1,2, \ldots, n\}$. Let $1<k<n$ and assume that for any $j_{1}, \ldots, j_{k} \in\{1,2, \ldots, n\}, j_{p} \neq j_{q}$, $p \neq q, \mathscr{V}_{k}:=\mathscr{V}\left(j_{1}, \ldots, j_{k}\right)$ is a Chebyshev subspace.

Suppose that there exist $1 \leq j_{1}<j_{2}<\cdots<j_{k}<j_{k+1} \leq n$ such that

$$
\mathscr{V}_{k+1}:=\mathscr{V}\left(j_{1}, \ldots, j_{k}, j_{k+1}\right)
$$

is a non-Chebyshev subspace. Without loss of generality we can assume that for any $k+1 \in\{1,2, \ldots, n\}, j_{m}=m, m=1,2, \ldots, k+1$. This means precisely that $V_{j_{m}}=\left[\left(V_{j_{m}}\right)_{i j}\right]_{i, j \in \mathrm{N}}$, where

$$
\left(V_{j_{m}}\right)_{i j}=\left\{\begin{array}{ll}
v_{i j_{m}}, & j=m \\
0, & j \neq m
\end{array} .\right.
$$

for $i \in \mathrm{N}, m \in\{1,2, \ldots, k, k+1\}$.

Since $\mathscr{V}_{k+1}$ is a non-Chebyshev subspace, there exists $A=\left[a_{i j}\right]_{i, j \in \mathrm{N}} \in$ $\mathscr{K}\left(c_{0}, c_{0}\right)$ such that $\sharp \mathscr{P}_{r_{k+1}}(A)>1$. We can assume that $0, W \in \mathscr{P}_{\mathscr{V}_{k+1}}(A)$, where $W \neq 0$. Let $\mathscr{U}=\left\{i:\left\|e_{i} \circ A\right\|=\|A\|\right\}$. Since $A \in \mathscr{K}\left(c_{0}, c_{0}\right), \sharp \mathscr{U}<\infty$. For every $i \in \mathscr{U}$ we put

$$
E_{i}=\left\{x \in \operatorname{ext} S_{l^{\infty}}:\left(e_{i} \otimes x\right)(A)=\|A\|\right\} .
$$

Since $0, W \in \mathscr{P}_{\gamma_{k+1}}(A)$, we conclude that for all $i \in \mathscr{U}$ and $x \in E_{i}$

$$
\left(e_{i} \otimes x\right)(W) \geq 0 .
$$

Let

$$
\mathscr{U}_{1}=\left\{i \in \mathscr{U}: \exists x \in E_{i}:\left(e_{i} \otimes x\right)(W)=0\right\} .
$$

Since $0 \in \mathscr{P}_{\mathscr{V}_{k+1}}(A), \mathscr{U}_{1} \neq \emptyset$.

We will prove that for any $i \in \mathscr{U}_{1}$ and $x, y \in E_{i}$ such that

$$
\begin{aligned}
\left(e_{i} \otimes x\right)(W) & =\left(e_{i} \otimes y\right)(W)=0, \\
x(l) & =y(l), \quad l=1,2, \ldots, k+1 .
\end{aligned}
$$

On the contrary, suppose that (6) does not hold. Let $x, y \in E_{i}$ be such that

$$
\left(e_{i} \otimes x\right)(W)=0, \quad\left(e_{i} \otimes y\right)(W)=0,
$$


and

$$
x(l) \neq y(l) \quad \text { for some } \quad l \in\{1,2, \ldots, k+1\} .
$$

Without loss of generality we can assume

$$
x(j)=y(j) \quad \text { for } \quad j=1,2, \ldots, p, p<k+1
$$

and

$$
x(j)=-y(j) \quad \text { for } \quad j=p+1, p+2, \ldots, k+1 .
$$

Hence

$$
\sum_{j=1}^{p} x(j) w_{i j}=0, \quad \sum_{j=p+1}^{k+1} x(j) w_{i j}=0 .
$$

As

$$
x(j)=-y(j) \quad \text { for } \quad j=p+1, p+2, \ldots, k+1
$$

we obtain

$$
a_{i j}=0 \quad \text { for } \quad j=p+1, p+2, \ldots, k+1 .
$$

By (5),

$$
\begin{aligned}
& \sum_{j=p+1}^{k} x(j) w_{i j}-x(k+1) w_{i, k+1} \geq 0 \\
& \sum_{j=p+1}^{k}-x(j) w_{i j}+x(k+1) w_{i, k+1} \geq 0 .
\end{aligned}
$$

Therefore

$$
\sum_{j=p+1}^{k} x(j) w_{i j}=x(k+1) w_{i, k+1} .
$$

By (7), $x(k+1) w_{i, k+1}=0$. Consequently, $w_{i, k+1}=0$. Hence $W \in \mathscr{V}_{k}$. Since $0 \in \mathscr{V}_{k}$ and $\mathscr{V}_{k}$ is a Chebyshev subspace, (6) is proved.

We will show that there exists $\alpha_{0}>0$ such that for every $\alpha \in\left(0, \alpha_{0}\right]$,

(8) $E(A-\alpha W)=\left\{e_{i} \otimes x: i \in \mathscr{U}_{1},\left(e_{i} \otimes x\right)(W)=0,\left(e_{i} \otimes x\right)(A)=\|A\|\right\}$.

We first prove that

(9) $\sup \left\{f(A): f=e_{i} \otimes x, i \in \mathscr{U}: f(W)<0\right\}$

$$
\leq\|A\|-2 \min \left\{\left|a_{i j}\right|: i \in \mathcal{U}, j \in\{1,2, \ldots, n\}, a_{i j} \neq 0\right\},
$$


where $A=\left[a_{i j}\right]_{i, j \in \mathrm{N}}$.

Let $i \in \mathcal{U}, f=e_{i} \otimes x, f(W)<0$. Hence there exists $j_{0} \in\{1,2, \ldots, n\}$ satisfying

$$
x\left(j_{0}\right) \neq \operatorname{sgn}\left(a_{i j_{0}}\right) \quad \text { for } \quad a_{i j_{0}} \neq 0 .
$$

Now, we will show

$$
\begin{aligned}
f(A)=\sum_{j=1}^{\infty} x & (j) a_{i j} \leq\|A\|-2\left|a_{i j_{0}}\right| \\
& \leq\|A\|-2 \min \left\{\left|a_{i j}\right|: i \in \mathcal{U}, j=1,2, \ldots, n,\left|a_{i j}\right| \neq 0\right\},
\end{aligned}
$$

and (9) is proved.

We conclude from (9) that there exist $\alpha_{0}>0, b>0$ such that for every $\alpha \in\left(0, \alpha_{0}\right]$,

$$
f(A-\alpha W)<b<\|A\|,
$$

where $f \in \operatorname{ext} S_{\mathscr{C}^{*}\left(c_{0}, c_{0}\right)}, f(W)<0$.

Assume $\alpha_{0}$ is so small that

$$
\sup _{i \in \mathbb{N} \backslash \mathscr{U}}\left\|e_{i} \circ\left(A-\alpha_{0} W\right)\right\|<\|A\| .
$$

Consequently, if $f \in E\left(A-\alpha_{0} W\right)$ then $f=e_{i} \otimes x$, where $i \in \mathscr{U}_{1}$ and $f(W)=0$. Since

$$
\left\|A-\alpha_{0} W\right\|=\|A\|=\operatorname{dist}\left(A, \mathscr{V}_{k+1}\right)
$$

(8) is proved.

Since $\alpha_{0} W \in \mathscr{P}_{\mathscr{V}_{k+1}}(A)$, we conclude (see [16]) that

$$
\exists 1 \leq q \leq k+2, \quad \exists \lambda_{1}, \ldots, \lambda_{q}>0, \quad \sum_{m=1}^{q} \lambda_{m}=1
$$

such that

$$
\left.\sum_{m=1}^{q} \lambda_{m}\left(e_{i_{m}} \otimes x^{i_{m}}\right)\right|_{\mathscr{V}_{k+1}}=0
$$

where $\left(e_{i_{m}} \otimes x^{i_{m}}\right)\left(A-\alpha_{0} W\right)=\left\|A-\alpha_{0} W\right\|$. Let $q$ be the smallest number having property (10). By (6), $i_{j} \neq i_{l}$ for $j \neq l, j, l \in\{1,2, \ldots, q\}$. If $q=k+2$ then (see [18]) $\alpha_{0} W$ is the strongly unique best approximation for $A$ in $\mathscr{V}_{k+1}$, a contradiction. Suppose that $1 \leq q \leq k+1$. This contradicts (ii). 
Let us assume that $\mathscr{V}_{k}$ is a Chebyshev subspace of $\mathscr{K}\left(c_{0}, c_{0}\right)$ for every $1 \leq k \leq n$. Suppose that (ii) is false. Consequently, there exist

$$
\begin{gathered}
1 \leq k \leq n, \quad 1 \leq j_{1}<j_{2}<\cdots<j_{k} \leq n, \\
1 \leq i_{1}<i_{2}<\cdots<i_{k}, \\
x_{m l} \in \mathrm{R}:\left|x_{m l}\right|=1, \quad m, l=1,2, \ldots, k
\end{gathered}
$$

satisfying

$$
\operatorname{det}\left[x_{m l} v_{i_{m} j_{l}}\right]_{m=1,2, \ldots, k, l=1,2, \ldots, k}=0 .
$$

It follows that there exist

$$
\lambda_{1}, \ldots, \lambda_{k} \in \mathrm{R}, \quad \sum_{m=1}^{k}\left|\lambda_{m}\right|>0
$$

such that

$$
\left.\sum_{m=1}^{k} \lambda_{m}\left(e_{i_{m}} \otimes x^{i_{m}}\right)\right|_{\mathscr{V}_{k}}=0
$$

where $x^{i_{m}}=\left(x^{i_{m}}(1), x^{i_{m}}(2), \ldots\right), x^{i_{m}}(l)=x_{m l}$.

Without loss of generality we can assume

$$
\lambda_{m}>0, \quad m=1,2, \ldots, k, \quad \sum_{m=1}^{k} \lambda_{m}=1 .
$$

We define an operator $B=\left[b_{i j}\right]_{i, j \in \mathrm{N}}$ by

$$
\begin{array}{rlrl}
b_{i j} & =\frac{\operatorname{sgn} x^{i}(j)}{2^{j}}, & & i \in\left\{i_{1}, i_{2}, \ldots, i_{k}\right\}, \\
b_{i j}=0, & & i \notin\left\{i_{1}, i_{2}, \ldots, i_{k}\right\}, \quad j \in \mathbf{N} .
\end{array}
$$

Hence $\left(e_{i_{m}} \otimes x^{i_{m}}\right)(B)=\|B\|, m=1,2, \ldots, k$. By (11), $0 \in \mathscr{P}_{\mathscr{V}_{k}}(B)$ and

$$
\operatorname{dim} \operatorname{span}\left\{e_{i_{m}} \otimes x^{i_{m}} \mid \mathscr{V}_{k}\right\}<k,
$$

where $\operatorname{dim} \mathscr{V}_{k}=k$. Therefore there exists $V \in \mathscr{V}_{k} \backslash\{0\}$ such that

$$
\left(e_{i_{m}} \otimes x^{i_{m}}\right)(V)=0, \quad m=1,2, \ldots, k .
$$

Note that (see the proof of the formula (9))

$$
\begin{aligned}
\sup \{f(B): f & \left.=e_{i_{m}} \otimes x, m=1,2, \ldots, k, f(V)<0\right\} \\
& <\|B\|-\min \left\{\left|b_{i j}\right|: i=i_{1}, i_{2}, \ldots, i_{k}, j=1,2, \ldots, n\right\} .
\end{aligned}
$$


Hence there exist $\alpha_{0}>0, b>0$ such that

$$
f\left(B-\alpha_{0} V\right) \leq b<\|B\|, \quad f \in \operatorname{ext} S_{\mathscr{K}^{*}}\left(c_{0}, c_{0}\right), \quad f(V) \leq 0 .
$$

Consequently, $\left\|B-\alpha_{0} V\right\|=\|B\|$, a contradiction. The proof is complete.

Example 3. We will construct an $n$-dimensional Chebyshev subspace $\mathscr{V} \subset$ $\mathscr{K}\left(c_{0}, c_{0}\right)$. Let $0<t_{1}<t_{2}<\cdots<t_{n-1}$ be such that

$$
\lim _{i \rightarrow \infty} \frac{1}{2^{i}} t_{m}^{i}=0, \quad m=1,2, \ldots, n-1 .
$$

Define $V_{m}=\left[\left(v_{m}\right)_{i j}\right]_{i, j \in \mathrm{N}}$ by

$$
\left(v_{m}\right)_{i m}=\frac{1}{2^{i}} t_{m}^{i}, \quad\left(v_{m}\right)_{i j}=0, \quad i \in \mathrm{N}, \quad j \neq m .
$$

Hence $V_{m} \in \mathscr{K}\left(c_{0}, c_{0}\right)$ for every $m=1,2, \ldots, n-1$.

Let $\mathscr{V}_{n-1}:=\operatorname{lin}\left\{V_{1}, V_{2}, \ldots, V_{n-1}\right\}$ satisfy the formula (ii) for every $1 \leq$ $k \leq n-1$.

We will construct an operator $V_{n} \in \mathscr{K}\left(c_{0}, c_{0}\right)$ such that $\mathscr{V}_{n}:=\operatorname{lin}\left\{V_{1}, V_{2}\right.$, $\left.\ldots, V_{n-1}, V_{n}\right\}$ satisfies the formula (ii) for every $1 \leq k \leq n$. Our goal is to find $x \in \mathrm{R}$ such that

$$
\lim _{i \rightarrow \infty} \frac{1}{2^{i}} x^{i}=0
$$

and

$$
\begin{aligned}
& W\left(x, y^{1}, \ldots, y^{k}, i_{1}, \ldots, i_{k}, m_{1}, \ldots m_{k-1}\right)
\end{aligned}
$$

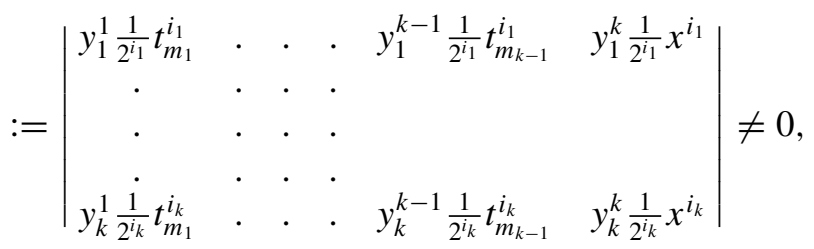

where $k \in\{1,2, \ldots, n\}, i_{1}, i_{2}, \ldots, i_{k} \in \mathrm{N}, y^{1}, \ldots, y^{k} \in\{-1,1\}^{k}, m_{1}, m_{2}$, $\ldots, m_{k-1} \in\{1,2, \ldots, n-1\}$. Since $W\left(x, y^{1}, \ldots, y^{k}, i_{1}, \ldots, i_{k}, m_{1}, \ldots\right.$, $\left.m_{k-1}\right)$ is not totally equal to zero, we conclude that the set of roots of $W\left(x, y^{1}\right.$, $\left.\ldots, y^{k}, i_{1}, \ldots, i_{k}, m_{1}, \ldots m_{k-1}\right)$ is finite for arbitrary but fixed $y^{1}, \ldots, y^{k}$, $i_{1}, \ldots, i_{k}, m_{1}, \ldots m_{k-1}$. Therefore for all $y^{1}, \ldots, y^{k}, i_{1}, \ldots, i_{k}, m_{1}, \ldots m_{k-1}$ as above, the set of roots of $W\left(x, y^{1}, \ldots, y^{k}, i_{1}, \ldots, i_{k}, m_{1}, \ldots m_{k-1}\right)$ is countable. Since R is not countable we see that there exists $x \in \mathrm{R}$ satisfying (12) and (13). 
Remark 2. An $n$-dimensional Chebyshev subspace proposed in Example 3 is a non-interpolating subspace of $\mathscr{K}\left(c_{0}, c_{0}\right)$.

Proof. Let us assume that $\mathscr{V}_{n}=\operatorname{lin}\left\{V_{1}, V_{2}, \ldots, V_{n}\right\}$ is an $n$-dimensional Chebyshev subspace, where $V_{m}, m=1,2, \ldots, n$ are defined in Example 3.

Put $V=\frac{1}{t_{1}} V_{1}-\frac{1}{t_{2}} V_{2}$. Note that $V \neq 0$ and $v_{i j}=0, j \geq 3, i \in \mathrm{N}$, where $V=\left[v_{i j}\right]_{i, j \in \mathrm{N}}$. It is obvious that there exist $x^{1}, x^{2}, \ldots, x^{n} \in \operatorname{ext} S_{l \infty}$ such that $x^{m}(1)=x^{m}(2)=1, m=1,2, \ldots, n$ and $f_{m}:=e_{1} \otimes x^{m}, m=1,2, \ldots, n$ are linearly independent. Note that

$$
f_{m}(V)=0, \quad m=1,2, \ldots, n .
$$

This completes the proof.

Lemma. Let $X$ be a normed space and let $\mathscr{V}$ be a finite-dimensional subspace of $X$. Let $T \in X$. If $0 \in \mathscr{P}_{\mathscr{V}}(T)$ and 0 is not a strongly unique best approximation for $T$ in $\mathscr{V}$ then

$$
\exists V \in \mathscr{V}, \quad V \neq 0 \quad: \quad \forall f \in E(T) \quad f(V) \geq 0 .
$$

Proof. Let us assume that

$$
\forall V \in \mathscr{V}, \quad V \neq 0, \quad \exists f \in E(T) \quad: \quad f(V)<0 .
$$

Set for any $V \in \mathscr{V},\|V\|=1$,

$$
\begin{aligned}
-r_{V} & =\inf \{f(V): f \in E(T)\}, \\
-r & =\sup \left\{-r_{V}: V \in \mathscr{V},\|V\|=1\right\} .
\end{aligned}
$$

We show that $r>0$. If not, there exists $\left(V_{n}\right) \subset S_{\mathscr{V}}$ such that $-r_{V_{n}} \geq-\frac{1}{n}$. Since $\mathscr{V}$ is a finite-dimensional subspace, we may assume that $V_{n} \rightarrow V \in S_{\mathscr{V}}$. Take $f \in E(T), f(V)<0$. Hence for $n \geq n_{0}$ there exists $d>0$ such that

$$
-\frac{1}{n} \leq-r_{V_{n}} \leq f\left(V_{n}\right)<f(V)+d<0,
$$

a contradiction. Therefore

$$
\forall V \in \mathscr{V}, \quad V \neq 0, \quad \exists f \in E(T) \quad: \quad f\left(\frac{V}{\|V\|}\right)<-r .
$$

By the above,

$$
\forall V \in \mathscr{V}, \quad V \neq 0, \quad \exists f \in E(T) \quad: \quad f(V) \leq-r\|V\| .
$$


Hence 0 is a strongly unique best approximation for $T$, a contradiction. This proves the lemma.

TheOREM 6. Let $\mathscr{V} \subset \mathscr{K}\left(c_{0}, c_{0}\right)$ be an $n$-dimensional subspace such that

$$
\forall V \in \mathscr{V}, \quad \forall i \in \mathrm{N} \quad \sharp\left\{j \in \mathrm{N}: v_{i j} \neq 0\right\}<\infty,
$$

where $V=\left[v_{i j}\right]_{i, j \in \mathrm{N}}$ and let $T \in \mathscr{K}\left(c_{0}, c_{0}\right)$. Then $T$ has a unique best approximation in $\mathscr{V}$ if and only if $T$ has a strongly unique best approximation in $\mathscr{V}$.

Proof. Let us assume that 0 is the unique best approximation for $T$ in $\mathscr{V}$. Suppose that 0 is not a strongly unique best approximation. Hence (see Lemma)

$$
\exists V \in \mathscr{V}, \quad V \neq 0 \quad: \quad \forall f \in E(T) \quad f(V) \geq 0,
$$

where $f=e_{i} \otimes x^{i}$ for some $x^{i} \in \operatorname{ext} S_{l^{\infty}}$.

Put

$$
\mathcal{N}=\left\{i \in \mathrm{N}: \exists x^{i} \in \operatorname{ext} S_{l^{\infty}}: e_{i} \otimes x^{i} \in E(T)\right\} .
$$

Since $T$ is compact, we conclude that $\sharp \mathscr{N}<\infty$.

For every $i \in \mathscr{N}$ we set

$$
E_{i}=\left\{x^{i} \in \operatorname{ext} S_{l_{\infty}}:\left(e_{i} \otimes x^{i}\right)(T)=\|T\|\right\} .
$$

Let $i \in \mathrm{N} \backslash \mathcal{N}$. Hence there exists $b>0$ such that

$$
\left(e_{i} \otimes x\right)(T)<b<\|T\|, \quad x \in \operatorname{ext} S_{l \infty} .
$$

Consequently, there exists $\alpha_{0}>0$ such that for every $\alpha \in\left(0, \alpha_{0}\right]$,

$$
\left|\left(e_{i} \otimes x\right)(T-\alpha V)\right|<b .
$$

Therefore

$$
\sup _{i \in \mathbf{N} \backslash \mathcal{N}}\left|\left(e_{i} \otimes x\right)(T-\alpha V)\right| \leq b<\|T\| .
$$

Let $i \in \mathscr{N}$ and let $x^{i} \notin E_{i}$. From this we conclude that there exists $j_{0} \in \mathbf{N}$ such that

$$
\operatorname{sgn} x^{i}\left(j_{0}\right) \neq \operatorname{sgn}\left(t_{i j_{0}}\right), \quad t_{i j_{0}} \neq 0,
$$

where $T=\left[t_{i j}\right]_{i, j \in \mathbf{N}}$.

Set $J=\left\{j \in \mathbf{N}: v_{i j} \neq 0\right\}$. If $\operatorname{sgn} x^{i}(j)=\operatorname{sgn}\left(t_{i j}\right)$ for any $j \in J$, then there exists $y^{i} \in E_{i}$ such that

$$
\left(e_{i} \otimes y^{i}\right)(T)=\|T\|, \quad\left(e_{i} \otimes y^{i}\right)(V)=\left(e_{i} \otimes x^{i}\right)(V) .
$$


By the above,

$$
\left(e_{i} \otimes x^{i}\right)(T-\alpha V) \leq\|T\|-\left(e_{i} \otimes y^{i}\right)(\alpha V) \leq\|T\| .
$$

Let $\operatorname{sgn} x^{i}\left(j_{0}\right) \neq \operatorname{sgn}\left(t_{i j_{0}}\right)$ for some $j_{0} \in J$, where $t_{i j_{0}} \neq 0$. Since $J$ is finite, there exists $\alpha_{0}>0$ such that

$$
\left\|\alpha_{0} V\right\|<\min \left\{\left|t_{i j}\right|: j \in J, t_{i j} \neq 0\right\}
$$

Let $\alpha \in\left(0, \alpha_{0}\right]$. Hence

$$
\begin{aligned}
\left(e_{i} \otimes x^{i}\right)(T-\alpha V) & =\sum_{j \in J} x^{i}(j)\left(t_{i j}-\alpha v_{i j}\right)+\sum_{j \notin J} x^{i}(j)\left(t_{i j}-\alpha v_{i j}\right) \\
& \leq \sum_{j \in J}\left|t_{i j}\right|-2\left|t_{i j_{0}}\right|+\sum_{j \notin J}\left|t_{i j}\right|+\alpha\|V\| \\
& =\|T\|+\alpha\|V\|-2\left|t_{i j_{0}}\right|<\|T\| .
\end{aligned}
$$

Finally,

$$
\|T-\alpha V\|=f(T-\alpha V),
$$

where $f=e_{i} \otimes x^{i}, i \in \mathcal{N}, x^{i} \in E_{i}$. Hence

$$
\|T-\alpha V\|=f(T-\alpha V) \leq\|T\| .
$$

The proof is complete.

AcKnowledgments. The author wishes to thank Professor Lewicki for his remarks and suggestions concerning this article.

\section{REFERENCES}

1. Aleksiewicz, A., Analiza funkcjonalna, PWN, Warszawa 1969.

2. Ault, D. A., Deutsch, F. R., Morris, P. D., Olson, J. E., Interpolating subspaces in approximation theory, J. Approx. Theory 3 (1970), 164-182.

3. Brosowski, B., Wegmann, R., Charakterisierung bester Approximationen in normierten Vektorräumen, J. Approx. Theory 3 (1970), 369-397.

4. Collins, H. S., Ruess, W., Weak compactness in spaces of compact operators and vector-valued functions, Pacific J. Math. 106 (1983), 45-71.

5. Deutsch, F., Li, W., Strong uniqueness, Lipschitz continuity and continuous selections for metric projections in $L_{1}$, J. Approx. Theory 66 (1991), 198-224.

6. Edwards, R. E., Functional Analysis. Theory and Applications, Dover, New York 1995.

7. Kowynia, J., Best approximation in finite dimensional Chebyshev subspaces of $L\left(l_{1}^{n}, c\right)$, East J. Approx. 12 (2006), 407-416.

8. Lewicki, G., Best approximation in finite-dimensional subspaces of $L(W, V)$, J. Approx. Theory 81 (1995), 151-165. 
9. Li, W., Strong uniqueness and Lipschitz continuity of metric projections: a generalization of the classical Haar theory, J. Approx. Theory 56 (1989), 164-184.

10. Malbrock, J., Chebyshev subspaces in the space of bounded linear operators from $c_{0}$ to $c_{0}$, J. Approx. Theory 9 (1973), 149-164.

11. Nürnberger, G., Strong unicity constants in Chebyshev approximation, pp. 144-154 in: Numerical Methods of Approximation Theory 8 (Proc. Oberwolfach 1986), Internat. Schriftenreihe Numer. Math. 81, Birkhäuser, Basel 1987.

12. Phelps, R. R., Čebyšev subspaces of finite dimension in $L_{1}$, Proc. Amer. Math. Soc. 17 (1966), 646-652.

13. Remez, E., General Computation Methods for Chebyshev Approximation, Atomic Energy, Voroneck 1957.

14. Rudin, W., Functional Analysis, McGraw-Hill, New York 1973.

15. Ruess, W. M., Stegall, C., Extreme points in duals of operator spaces, Math. Ann. 261 (1982), 535-546.

16. Singer, I., Best Approximation in Normed Linear Spaces by Elements of Linear Subspaces, Grundlehren math. Wiss. 171, Springer, Berlin 1970.

17. Wójcik, A., Characterization of strong unicity by tangent cones, pp. 854-866 in: Z. Ciesielski (ed.), Approximation and Function Spaces (Proc. Gdańsk 1979), PWN, Warszawa/NorthHolland, Amsterdam 1981.

18. Sudolski, J., Wójcik, A., Some remarks on strong uniqueness of best approximation, J. Approx. Theory Appl. 6 (1990), 44-78.

DEPARTMENT OF MATHEMATICS

AGH UNIVERSITY OF SCIENCE AND TECHNOLOGY

AL. MICKIEWICZA 30

30-059 CRACOW

POLAND

E-mail: joannakowynia@tlen.pl 